\title{
A Review Paper on Video Dehazing
}

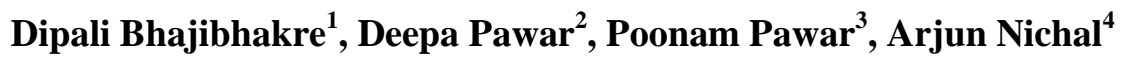 \\ BE Student, Electronics \& Telecommunication Engineering, AITRC, Vita, India ${ }^{1,2,3}$ \\ Assistant Professor, Electronics \& Telecommunication Engineering, AITRC, Vita, India ${ }^{4}$
}

\begin{abstract}
We make an investigation of dehazing effect of image and video which is affected by bad weather conditions. A video captured in outdoor scenes affects by presence of haze like fog, mist and dust particles in the atmosphere. We are utilizing a Dehazing algorithm to remove this unwanted haze from videos and Real-time video. For this we uses a Novel method of video dehazing based on contrast enhancement. From our observation it is conclude that hazy image and video has low contrast, so we restore the hazy image and video by enhancing its contrast. This algorithm computes the airlight on an input hazy image and video then we estimates the transmission map to maximize the contrast of output video and image. We establish a better Dehazing performance with fewer artifacts and better coding efficiency and demonstrate that the proposed algorithm can remove haze efficiently and recover the parameters of original scene.
\end{abstract}

Keywords: Real-time video dehazing, image dehazing, restoration, contrast enhancement, airlight estimation, transmission map,gamma correction.

\section{INTRODUCTION}

Recently there has been growing interest in analysis of video affected by weather phenomenon. Haze removal (Dehazing) is highly desired in consumer, computational photography and computer vision applications. The process of removing haze can significantly increase the visibility of scene and correct the shift caused by the airlight. Video captured in poor environmental condition fails to present visual effectively. Dehazing is the process of removing haze from video and enhancing the video quality so; the main object of our technique is to enhance the poor visibility of the video, which applicable in the field of public safety, traffic accident analysis, crime forensics, remote sensing area and military surveillance. The three main objectives of video enhancement techniques are, to explore the hidden details in the video; the effect such as flickering and uneven exposure should be avoided; The video should be temporally consistent. To achieve all this objectives we propose a contrast enhancement algorithm. Contrast Enhancement commonly used for surveillance applications because the viewing environment is outside the control of the observer. By using this method, unexpected flickers are effectively eliminated. Optical scattering produces an unnecessary exposure on a video and image which known as 'airlight'.It happens because of light coming from the source (sun) is scattered to words the observer. The airlight is firstly estimated in given input scenes (video, image).To compute the scene depth there are several approaches for example scene depths are estimated from two or more image and video which are captured in different environmental and weather conditions. For this we divide an input scene into multiply blocks and then estimate the optimal transmission for that block's. So, the contrast of image and video increased. Finally we apply gamma correction technique which is used to optimize the usage of bits encoding an image and video. It is an effective tool for manipulating the histogram of an image that is either over an under exposed. In addition to manual control, gamma correction can be also automatically adjusted to compensate for change in the scene. In analog video system, gamma correction is performed with analog circuitry and is adjusted manually. With digital video system, gamma correction can be provided using mathematical operations in a digital circuitry. Thus we dehaze an image and video using contrast enhancement algorithm from hazy video and image to achieve haze free image and video which is clearly visible for human eyes and greatly impact the accuracy of the message and visual perception.

\section{BASIC METHDOLOGY}

In this paper we proposed a methodology that improves the visibility of haze image and video. The Methodology uses the depth estimation concept to restore the degraded image and video. The input image and video which are captured in the outdor scenes subjected to atmospheric troubles such as haze, fog and heavy rain etc. 


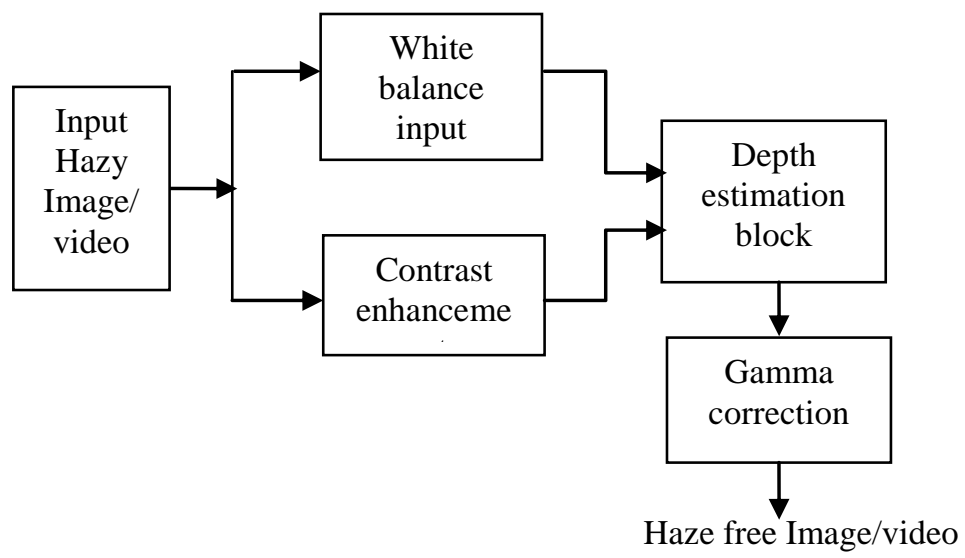

Figure 1. General Block Diagram of Video Dehazing

In order to identify the color temperature and the contrast levels the input image and video is converted into the two individual inputs such as white balance input and contrast enhancement. White balance basically means color balance. It is a function that gives the camera reflection to 'true white'. Since white light is sum of all other colors. The camera will then display all colors correctly. Contrast enhancement process is used to make image and video features more clear. Contrast manipulation involves a scene in oredr to increase the contrast. These two individual image and video are then applied to depth estimation process involves various segments such as finding the weight maps of individual image and video (for both white balance and contrast enhancement), normalization of weight maps and application of pyradmids.

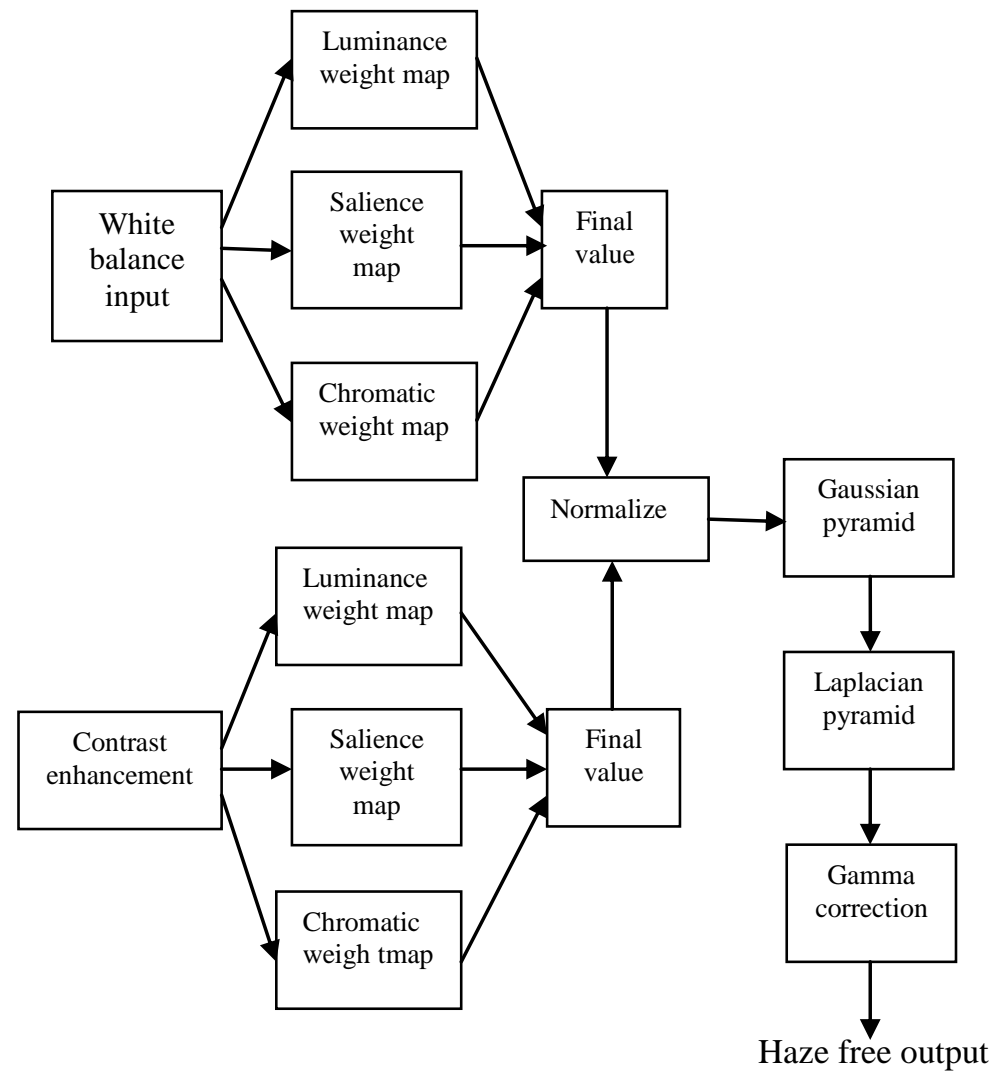

Figure 2. Depth Estimation Process 


\title{
International Journal of Advanced Research in Computer and Communication Engineering
}

\author{
Vol. 8, Issue 2, February 2019
}

A gamma correction factor has been applied to the depth estimation process in order to improve visibility which is perfect scene to human eye. The gamma correction enhance contrast level of the image and video and hence even in the high atmospheric troubles we get a visually perfect image and video. Finally we obtain a haze free image and video which clearly visible for human eye and greatly impact the accuracy of the message and visual perception.

Depth Estimation Process: Chromatic weight map controls the saturation gain in image and video, the Salience weight maps defines the quality whuch contributes to degress of conspicuousness with respect to the neighdorhood region. The output pf each weight maps are normalized (change the range of pixel intensity value), and then applied to Gaussian pyramid of length five. The image and video is a data structure designed to support efficient scaled convolution through reduced image and video representation. It consist of a sequence of copies of an original image and video in which both sample density and resolution are described in regular steps. The Laplacian pyramid has been described as a data structure composed of band pass copies of an image and video that is well suited for scaled image and video analysis. But the pyramid may also be viewed as an image and video transformation, or code. The pyramid nodes are then considered code elements, and the equivalent weight function are sampling function that give node values when convolved with the image and video.

\section{III.LITERATURE REVIEW}

A. In 1998, C. Tomasi, R. Manduchi, [1] proposed a Bilateral filtering for gray and color images. Bilateral filtering smooth's image while preserving edges, by means of a nonlinear combination of nearby image value. It combines, gray levels of color based both geometric closeness and their photometric similarity, and prefers near values to distant values in both domain and range. Filtering is perhaps the most fundamental operation of image processing and computer vision. The intuitions of that image typically vary slowly over space, so pixels are likely to have similar values, and it therefore appropriate to average them together. In this paper a non iterative scheme for edge preserving smoothing that is non iterative and simple. It allows explicitly enforcement of any desired notation of photometric distance, which is particularly important for filtering color images. It combines is much more interesting, which is denoted as bilateral filtering. Bilateral filtering can be applied to color image just. The CIE-lab color space endows the space of colors with short Euclidian distance correlate strongly with human color discrimination performance. Here only perceptually similar color is averaged together; only perceptually visible edges are preserved.

B. In 2001, Y. Y. Schechner, G.Narsimhan and Shree K. Nayar [2] proposed a technique of Instant dehazing of image using polarization. They present a method which removes the haze from images which is affected by atmospheric particles, which is polarized. They obtained the hazed image and scene structure information through a model which is affected by polarization effects of atmospheric scattering. Because of air molecules and small dust particles polarization effects are explores, to remove this effect image is captured through polarizing filter which is settled parallel to plane. They obtained the range map of images. In that, darker points are used to indicate more instant objects. This will becomes helpful in the field of photography and remote sensing. But this method is less effective in the case of overcast sky and in presence of very dense haze or fog.

C. In 2003, S. G. Narasimhan and Shree K.Nayar, proposed a [3] Contrast Restoration of Weather Degraded Images. In this paper present a physical based model in the uniform bad weather conditions. Change in intensities of present scene point under the bad weather conditions provides the simple constraint to detect depth discontinuities in the scene. And also compute the scene structure then a fast algorithm to restore scene contrast is present. All the method described in this paper are effective under a wide range of weather conditions in which including haze, fog, mist. Further this method can be applied to gray scale, RGB color, multispectral and IR images. This method is not applicable to real time to obtain weather free video. This method cannot be used to restore the contrast of moving object or video. In which used the off-the-shelf 8bit digital video camera.

D. In 2006, S. Shwartz, E. Namer, Y. Y. Schechner published a paper on [4] Blind Haze Separation This paper proposes an approach for blind recovering parameters needed for separating the airlight from the measurement, thus recovering the contrast with neither user interaction nor existence of the sky in the frame for analyzing haze images, an effective approaches is based on polarization. Which measures pixels that correspond to sky by the horizon, thus estimation relies on the existence of such image part in the field of view (FOV).This method blindly separate the airlight radiance from the object signal it works even if no sky exists in the FOV. The method exploits mathematical tools developed in the field of Blind Source Separation (BSS), which also known as independent component analysis (ICA) which is based on color cues. 


\title{
International Journal of Advanced Research in Computer and Communication Engineering
}

\author{
Vol. 8, Issue 2, February 2019
}

Here obtaining the blind parameter estimation which was consistent with direct sky measurement, consequently, dehazing showed significant improvement of visible and color reactive to the raw data.

E. In 2007, J. P. Oakely and H. Bu proposed [5] a Correction of simple contrast loss in color image. An algorithm is proposed which described for estimating the level the airlight given the assumption that it is constant throughout the image. It finds the minimum of a global cost function and is applicable to both monochrome and color image. The performance of the algorithm is explored using the Monet Carlo simulation with synthetic images under different statistical assumptions. The outdoor scene of image will often degraded often be optical scattering of light caused due to fog or mist which produces additional lightness present in some part of image, this effect is referred as atmospheric background radiation or airlight. In this paper mitigation of simple contrast loss of image is happened, which caused by parameters added in the image while captured by the camera. In simple contrast loss, the degradation by described. A physical based method is proposed to restore simple contrast loss due to a scattering medium and other source of light. The first method provides accurate contrast restoration of color image in the scene that processed foggy image is similar to one taken in clear condition. A method has been proposed for determination of airlight level in digital images, which involves the minimization of a scalar global cost function and no region segmentation is required and simple contrast loss is easily corrected. The accuracy of the method under ideal conditions has been confirmed with synthetic image model. The method is applicable to both black and white image, color images.

F. In 2008, Robby T. Tan published a paper on [6] Visibility in Bad Weather from a Single Image. This paper proposes an automated method that only requires a single input image, which is based on two basic observations; First, image with enhanced visibility (or color-day) have more contrast than image plugged by bad weather. Second airlight, whose variation mainly depends on the distance of objects to viewer, tends to be smooth. This method does not require the geometrical information of input image and it is applicable for both color and gray images. In bad weather conditions poor visibility is a major problem for many applications of computer version, which are most automatic systems for surveillance, intelligent vehicles, outdoor object recognition etc. The light from the atmosphere and from the object are observed and scattered by those particles, causing the visibility of scene to be degraded. In this method an input image is given which estimate the atmospheric light from which we obtain the light chromatically. Then the light color remove from the input image and the data is computed. So this paper proposes a method that is solely based on single images without requiring the geometrical structure of neither the world nor any user interactions. So, the method is proposed that is solely based on single image without requiring the geometrical structure of world nor any user interactions. This is applicable for outdoor surveillance system, intelligent vehicle system, remote sensing system, graphics editors, etc.

G. In 2009, K. He, J. Sun, X. Tang [7] proposed a paper on Single image haze removal using dark channel prior method. In this project, they removed the haze from single image using dark channel prior method. By using this method, they obtained haze thickness and so get an high quality haze free image. Images are mainly absorption and scattering. Because of this reason, image losses its contrast and color fidelity. So, by clearing this effect of atmospheric i.e., haze the image will visible clearly and increases its contrast. They enhanced the contrast of restored image. This method is also applicable to distant object which is in heavy haze. But, it similar to airlight in a large region. They used 3D models and texture maps of image to remove the haze from image.

H. In 2011, J-Hwan, J-Y Sim, C-Su, Kim, [8] proposed a Single image dehazing based on contrast enhancement. An algorithm is proposed on single image using contrast enhancement. This algorithm is applied on single hazy image. The first step is airlight estimation which is based on quad-tree subdivision. Next, estimation of optimal transmission is done to enhance the contrast of restore image. Ambient light in atmosphere is nothing but airlight. It is considered as brightest color in image. They used hierarchal method based on quad-tree subdivision to estimate airlight. The algorithm estimates spacevarying transmission value. For that input image is divided into many block is estimated in order to enhance the contrast of image. By developing low complexity algorithm we can apply it on video also as it requires more power than single image.

I. In 2016, Feng Yu, Chunmei Qing, Xiangmin Xu, BolunCai, proposed a [9] Image and video dehazing using Viewbased cluster segmentation. A view-based cluster segmentation method is utilized to avoid distortion in sky regions and make the sky and white objects clear. Here firstly GMM (Gaussian Mixture Model) is utilized to cluster the depth based on distant view to estimate the sky region and distant view to estimate is modified to reduce distortion. Secondly, Single haze image is divided into 'K- classifications', finally, online GMM cluster is applied to video dehazing. Here this method is proposed by using GMM cluster and color attenuation prior for the depth map and the haze image separately the transmission estimation and atmospheric light estimation and modified using the view based cluster segmentation to decrease color distortion and improve global contrast; video dehazing algorithm is presented by using online GMM cluster. 


\title{
International Journal of Advanced Research in Computer and Communication Engineering
}

\author{
Vol. 8, Issue 2, February 2019
}

The global brightness of sequence dehaze image is increased and finally video dehazing method is proposed, which can restore hazy video by saving a lot of time for the cluster of sequence of video.

J. In 2018, Yongmin Park, Tae-Hwan Kim, proposed [10] a Fast execution scheme for dark-channel-prior based outdoor video dehazing. It realizes the fast execution of the dark-channel-prior method targeting the outdoor video dehazing. The overall execution time is reduced by up to $49 \%$ while dehazing quality is maintained to that of original method. The signal processing used to remove haze is called dehazing. Density of the haze of image is non uniform for every pixel of image. So detecting the dark pixel of image removes its haze. Camera captures the hazy image and detects the location of airlight. To measure the contrast develops a cost function, which consist of standard deviation term and histogram uniform term. Finally experimental result demonstrates that the proposed algorithm can remove haze as well as reconstruct the details in the original scenes more clearly.

Comparison of all above papers is shown in Appendix -1

\section{IV.CONCLUSION}

Analysis of Video and Image affected by weather phenomenon and environmental conditions (nothing but Haze), an algorithm is proposed as, 'Contrast Enhancement'. Contrast enhancement is a process that makes the video and image features stand out more clearly by making optimal use of the colours available on the display or output device. This algorithm is applied to the Image and video and then we moving towards the Real-time video, which can be used in surveillance system, in the field of Public safety. Video and Image quality can be clear by estimating the airlight. Then, the depth estimation process is used to identify the depth information of given input. Finally gamma correction technique is used to clear the quality or visual perception of image and video. Hence finally we have a result of pure, clear image and video which is visible to human eye.

\section{Appendix -1}

\begin{tabular}{|l|l|l|l|l|}
\hline Author \& year & Paper title & Technique used & Advantages & Disadvantage \\
\hline $\begin{array}{l}\text { C. Tomasi, R. Manduchi, } \\
1998\end{array}$ & $\begin{array}{l}\text { Bilateral Filtering } \\
\text { for gray and color } \\
\text { images }\end{array}$ & $\begin{array}{l}\text { Bilateral filtering } \\
\text { and non iterative }\end{array}$ & $\begin{array}{l}\text { Applicable for } \\
\text { images }\end{array}$ & $\begin{array}{l}\text { Not applicable for } \\
\text { black-and-white } \\
\text { ones. }\end{array}$ \\
\hline $\begin{array}{l}\text { Y. Y. Schechner, S. G. } \\
\text { Narsimhan, S. K. Nayar, } \\
2001\end{array}$ & $\begin{array}{l}\text { Instant dehazing } \\
\text { image using } \\
\text { polarization }\end{array}$ & $\begin{array}{l}\text { Polarization } \\
\text { technique }\end{array}$ & $\begin{array}{l}\text { Helpful in the field } \\
\text { of photography and } \\
\text { remote sensing }\end{array}$ & $\begin{array}{l}\text { This method is less } \\
\text { effective in the case } \\
\text { of overcast sky and } \\
\text { in presence of very } \\
\text { dense haze or fog }\end{array}$ \\
\hline $\begin{array}{l}\text { S. G. Narsimhan, S. K. } \\
\text { Nayar, 2003 }\end{array}$ & $\begin{array}{l}\text { Contrast restoration } \\
\text { weather degraded } \\
\text { images }\end{array}$ & $\begin{array}{l}\text { Physical- based } \\
\text { method to recover } \\
\text { blind parameter }\end{array}$ & $\begin{array}{l}\text { The method is } \\
\text { applicable for gray } \\
\text { scale, RGB color, } \\
\text { multispectral, IR } \\
\text { image }\end{array}$ & $\begin{array}{l}\text { Problem of restoring } \\
\text { the image the } \\
\text { contrast of } \\
\text { automatically } \\
\text { degraded image }\end{array}$ \\
\hline $\begin{array}{l}\text { S. Shwartz, E. Namer, Y. } \\
\text { Y. Schechner, 2006 }\end{array}$ & $\begin{array}{l}\text { Blind haze } \\
\text { separation }\end{array}$ & $\begin{array}{l}\text { Polarization } \\
\text { technique }\end{array}$ & $\begin{array}{l}\text { Dehazing showed } \\
\text { significant } \\
\text { improvement of } \\
\text { visibility and color, } \\
\text { reactive to the raw } \\
\text { data }\end{array}$ & $\begin{array}{l}\text { Problem of restoring } \\
\text { the contrast of } \\
\text { atmospherically } \\
\text { degraded image and } \\
\text { video. }\end{array}$ \\
\hline
\end{tabular}




\section{International Journal of Advanced Research in Computer and Communication Engineering}

Vol. 8, Issue 2, February 2019

\begin{tabular}{|c|c|c|c|c|}
\hline Author \& year & Paper title & Technique used & Advantages & Disadvantage \\
\hline J. P. Oakley, H. Bu, 2007 & $\begin{array}{l}\text { Correction of simple } \\
\text { contrast loss in } \\
\text { color image }\end{array}$ & $\begin{array}{l}\text { Physical based } \\
\text { method }\end{array}$ & $\begin{array}{l}\text { This method is } \\
\text { applicable for both } \\
\text { black and white and } \\
\text { color images }\end{array}$ & $\begin{array}{l}\text { Considered the } \\
\text { bright parts of } \\
\text { image, hence this } \\
\text { algorithm does not } \\
\text { give good result }\end{array}$ \\
\hline R. Fattal, 2008 & $\begin{array}{l}\text { Single image } \\
\text { dehazing }\end{array}$ & Automated method & $\begin{array}{l}\text { Solely based on } \\
\text { single images, } \\
\text { without requiring } \\
\text { the geometrical } \\
\text { structure }\end{array}$ & $\begin{array}{l}\text { There are some } \\
\text { halos surrounding } \\
\text { the trees in the } \\
\text { image. }\end{array}$ \\
\hline $\begin{array}{l}\text { K. He, J. Sun, X. Tang, } \\
2009\end{array}$ & $\begin{array}{l}\text { Single image haze } \\
\text { removal using dark } \\
\text { channel prior }\end{array}$ & $\begin{array}{l}\text { Dark-channel-prior } \\
\text { method }\end{array}$ & $\begin{array}{l}\text { To remove haze } \\
\text { from single input } \\
\text { image dark-channel- } \\
\text { prior method is } \\
\text { proposed }\end{array}$ & $\begin{array}{l}\text { Incapable for sun } \\
\text { influence in sky } \\
\text { region and bluish } \\
\text { hue near the horizon }\end{array}$ \\
\hline $\begin{array}{l}\text { Jin-Hwan Kim, J-Y Sim, } \\
\text { C-Su Kim, } 2011\end{array}$ & $\begin{array}{l}\text { Single image } \\
\text { dehazing based on } \\
\text { contrast } \\
\text { enhancement }\end{array}$ & $\begin{array}{l}\text { Contrast } \\
\text { enhancement }\end{array}$ & $\begin{array}{l}\text { To estimate a space } \\
\text { varying } \\
\text { transmission map to } \\
\text { dehaze an image }\end{array}$ & ---- \\
\hline $\begin{array}{l}\text { Feng Yu, Chunmei Qing, } \\
\text { Xiangmin Xu, Bolun Cai, } \\
2016\end{array}$ & $\begin{array}{l}\text { Image and video } \\
\text { dehazing using } \\
\text { view-based cluster } \\
\text { segmentation }\end{array}$ & $\begin{array}{l}\text { View-based cluster } \\
\text { segmentation }\end{array}$ & $\begin{array}{l}\text { Avoid the color } \\
\text { distortion in sky } \\
\text { region and make the } \\
\text { sky and white object } \\
\text { be clear }\end{array}$ & $\begin{array}{l}\text { If static analysis } \\
\text { method is used to } \\
\text { dehaze the video, } \\
\text { then it will take a lot } \\
\text { of time to process }\end{array}$ \\
\hline $\begin{array}{l}\text { Yongmin Park, Tae-Hwan } \\
\text { Kim, } 2018\end{array}$ & $\begin{array}{l}\text { Fast execution } \\
\text { scheme for dark- } \\
\text { channel-prior based } \\
\text { outdoor video } \\
\text { dehazing }\end{array}$ & $\begin{array}{l}\text { Dark-channel-prior } \\
\text { method }\end{array}$ & $\begin{array}{l}\text { Realize a fast } \\
\text { dehazing system } \\
\text { targeting outdoor } \\
\text { video streams } \\
\end{array}$ & ---- \\
\hline
\end{tabular}

\section{REFERENCES}

[1] C. Tomasi, R. Manduchi, "Bilatral filtering for gray and color images", In Proc. IEEE ICCV, pp. 839-846, January, 1998

[2] Y. Y. Schechner, S. K. Nayar, "Instant dehazing o image using polarization", In Proc. IEEE CVPR, vol. 1, pp. 325-332, December 2001.

[3] S. G. Narsimhan, S. K. Nayar, "Contrast restoration of weather degraded images”, IEEE Trans. Pattern Anal. Mach. Intell., Vol. 25, pp. 713-724, June 2003.

[4] S. Shwartz, E. Namar, Y. Y. Schechner, "Blind Haze separation”, IEEE CVPR vol. 2, pp. 1984-1991, June 2006.

[5] J. P. Oakley, H. Bu, "Correction of simple contrast loss in color image”, IEEE Trans. Image Process., vol. 16, pp. 512-522, Feb, 2007.

[6] R. Fattal, "Single image dehazing", ACM Trans. Graph, vol. 27, no. 3, pp, 1-9, August, 2008.

[7] K. He, J. Sun, X. Tang, "Single image Haze removal using dark channel prior ”, IEEE CVPR, pp. 1956-1963, June 2009.

[8] Jin-Hwan Kim, J-Y Sim, X. Tang, "Single image dehazing based on contrast enhancement", IEEE, International Conference on Acouatics, vol. 7882, no. 1, pp. 1273-1276, 2011.

[9] Feng Yu, Chunmei Qing, Xiangmin, Xu, Bolun Cai, “Image and video dehazing using view-based cluster segmentation”, 2016.

[10] Yongmin Park, Tae-Hwan kim, "Fast execution scheme for dark-channel-prior outdoor video dehazing", IEEE, ACCESS, vol. 6, pp. 10003-10014, March 2018.

[11] M. Gopika, M. Sirisha, "Visibility Enhancement of hazy image using Depth Estimation Concept”, IRJET, vol. 4, Issue. 7, pp. 3300 - 3305 , July 2017. 
International Journal of Advanced Research in Computer and Communication Engineering

Vol. 8, Issue 2, February 2019

\section{BIOGRAPHIES}

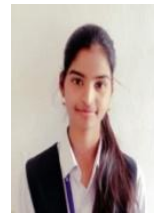

\section{Dipali Bhajibhakre}

Pursuing her BE in Electronics \& Telecommunication from Adarsh Institute of Technology \& Research Centre, Vita. Her area of interest is Image processing and Embedded system.

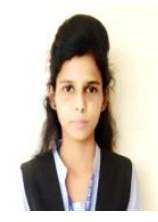

\section{Deepa Pawar}

Pursuing her BE in Electronics \& Telecommunication from Adarsh Institute of Technology \& Research Centre, Vita. Her area of interest is Image processing and Embedded system.

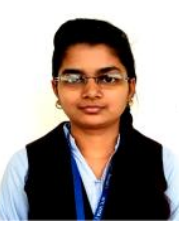

\section{Poonam Pawar}

Pursuing her BE in Electronics \& Telecommunication from Adarsh Institute of Technology \& Research Centre, Vita. Her area of interest is Image processing and Embedded system.

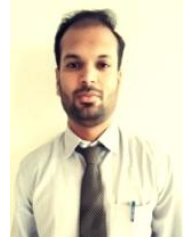

\section{Arjun Nichal}

Received his M.Tech degree from Walchand college of Engineering Sangli in 2012. Pursing Ph. D. from Shivaji University Kolhapur. Working as an Assistant Professor in Adarsh Institute of Technology \& Research Centre, Vita. His area of interest is Image processing and Embedded system. Published one E-book and 19 international journal papers. He has one blog on Fundamentals of Image Processing, Matlab Basics and Embedded System. Blog: www.imagelpcmatlab.blogspot.in 